\title{
Multicarrier CDMA Overlay for Ultra-Wideband Communications
}

\author{
Jiangzhou Wang, Senior Member, IEEE, and Laurence B. Milstein, Fellow, IEEE
}

\begin{abstract}
In this letter, the performance of multicarrier code-division multiple-access (CDMA) systems is studied in the presence of narrowband interference for future ultra-wideband (UWB) communications. A Nakagami fading channel is assumed, and notch filters along with diversity techniques are used in the multicarrier CDMA receiver. A complete performance analysis of error probability is given. It is shown that when the number of subcarriers jammed by narrowband interference is small, the multicarrier receiver without notch filters can work well, due to the gain of frequency diversity from nonjammed subcarriers. On the other hand, when the number of subcarriers jammed by the narrowband interference is large, using notch filters can improve the multicarrier system performance significantly.
\end{abstract}

Index Terms-Frequency diversity, multicarrier code-division multiple access (CDMA), Nakagami fading, RAKE receiver, ultra-wideband (UWB) communications.

\section{INTRODUCTION}

$\mathbf{R}$ ADIO spectrum is a limited and increasingly valuable resource. Recently, there has been a growing interest in the research and development of novel technologies aimed at allowing new services to use spectrum already allocated to established services, but without causing noticeable interference to the existing users. Ultra-wideband (UWB) systems [1], [2], using bandwidths in excess of $500 \mathrm{MHz}$ with very low power spectral density (PSD), are currently attracting much interest as a means of getting additional capacity by overlaying narrowband signals that currently occupy various portions of the spectrum. If the emissions from UWB devices are regulated to avoid causing significant interference to licensed narrowband services, then it becomes possible to allow UWB systems to operate on an unlicensed basis, enabling UWB technology to support a diverse range of short-distance applications, such as wideband multimedia services for the home, radar, automotive systems, and medical imaging systems.

Impulse radio [3] does not use a sinusoidal carrier to shift the signal to a frequency band in which signals propagate well, but instead communicates with a baseband signal composed of subnanosecond pulses (referred to as monocycles). Therefore, its bandwidth ranges from near dc to several gigahertz. The main advantage of impulse radio is that it does not need radio frequency (RF) components in transceivers. Therefore, implemen-

Paper approved by M. Chiani, the Editor for Transmission Systems of the IEEE Communications Society. Manuscript received December 20, 2002; revised December 15, 2003. This work was supported in part by Intel Corporation, in part by the Center for Wireless Communications at UCSD, in part by the CoRe Program of the State of California, and in part by the Research Grants Council of the Hong Kong SAR government.

$\mathrm{J}$. Wang is with the Department of Electrical and Electronic Engineering, University of Hong Kong, Hong Kong (e-mail: jwang@eee.hku.hk).

L. B. Milstein is with the Department of Electrical and Computer Engineering, University of California at San Diego, La Jolla, CA 92093 USA (e-mail: milstein@ece.ucsd.edu).

Digital Object Identifier 10.1109/TCOMM.2004.836448 tation is relatively simple and cost is low. However, this technology has one main disadvantage: its spectrum contains a lot of spectral lines. In other words, the signal power is mainly concentrated with spikes in the frequency domain, which makes overlay with narrowband systems difficult.

Direct-sequence (DS)-CDMA was proposed to co-exist with narrowband systems (a so-called CDMA overlay) about a decade ago [4]. It has been shown in [5] that in CDMA overlay situations, narrowband interference can be significantly suppressed using a notch filter, assuming the bandwidth of the narrowband interference is much smaller than that of CDMA signals. However, this single-carrier DS-CDMA overlay does not appear to be feasible when applied directly to UWB systems, due to the very large UWB bandwidth. In single-carrier DS, large bandwidth means high chip rate, which leads to too large a sampling rate for the analog-to-digital converter (A/D) in the receiver. Furthermore, when there are many narrowband interferers present, the envelope of the received composite signal exhibits large variation. Therefore, a very large dynamic range of the $\mathrm{A} / \mathrm{D}$ is required.

The multicarrier CDMA system proposed in [6] modulates the same data on different subcarriers. All subcarrier spectra are disjoint in frequency. One of the main advantages of multicarrier CDMA is that relatively low-speed A/Ds are used, with one for each subcarrier signal. In addition, the multicarrier CDMA not only allows for the use of RAKE on each subcarrier, but also has an inherent frequency-diversity capability by combining the outputs of the different subcarrier signals at the receiver. Moreover, it yields effective narrowband interference rejection in an overlay mode. For example, when there is a strong narrowband interferer in one of the subbands, in the worst case, the receiver can simply ignore the signal in that subcarrier band. Finally, in the absence of narrowband interference, both multicarrier and single-carrier CDMA have comparable multiaccess capability for a given system bandwidth. However, when multicarrier CDMA is applied to UWB, there are two important issues which need to be addressed.

1) The bandwidth of each subcarrier signal could be extremely wide with respect to that of the narrowband interference. Simply discarding a subcarrier that is experiencing narrowband interference might be too inefficient. A more effective way is to use a notch filter to suppress narrowband interference in each subcarrier. Then, even a jammed subcarrier signal can still make a positive contribution to the net frequency diversity.

2) The channel model used in [6] is too simple. It was assumed in [6] that the channel in each subcarrier only has one resolvable path, and flat Rayleigh fading was assumed for each subcarrier. However, the measurement results in [7] have shown that the UWB channel is a dense multipath channel. The number of resolvable paths could be in the dozens. In addition, the fading of each path is likely to 


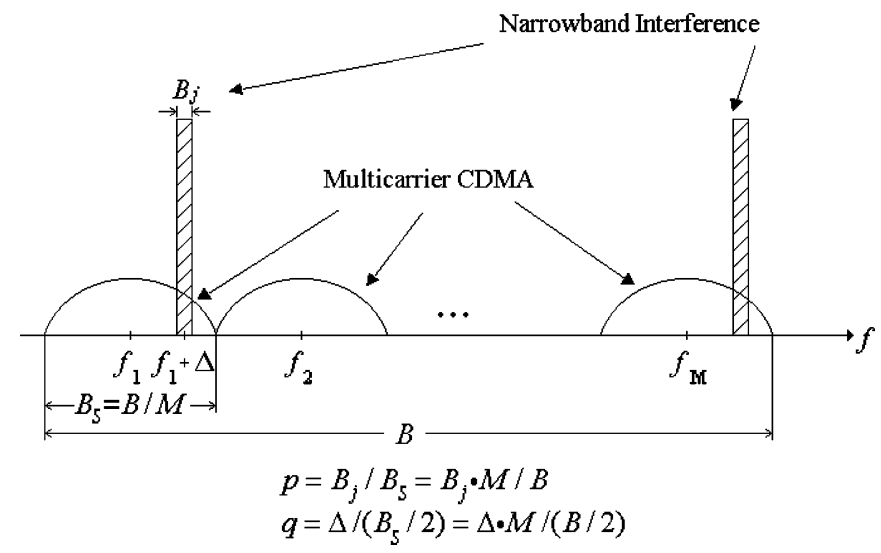

Fig. 1. Spectral densities of multicarrier CDMA and narrowband interference.

follow the gamma (or Nakagami) distribution, instead of a Rayleigh distribution. Thus, like single-carrier CDMA, a RAKE receiver can be used in each subcarrier to enhance performance [8]-[10].

Based on the above considerations, the objective of this letter is to study the performance of multicarrier DS-CDMA with a notch filter available in each subcarrier for future UWB communications over a highly dense multipath fading channel.

\section{SYSTEM MODELS}

The multicarrier CDMA overlay situation is shown in Fig. 1. Note that the spectra of different subcarriers in the multicarrier waveform are disjoint. It is assumed that the total bandwidth of the UWB system is $B$. Therefore, the bandwidth of each subcarrier is $B_{s}=B / M$, where $M$ is the number of subcarriers. For simplicity, we assume at most one narrowband interferer per subcarrier. The total number of narrowband interferers is $M_{1}\left(M_{1} \leq M\right)$. The parameters $p$ and $q$ are defined as the ratio of the bandwidth $\left(B_{j}\right)$ of a narrowband interferer to the bandwidth of one subcarrier, and the ratio of the offset $(\Delta)$ of the center frequency of a narrowband interferer from the $m$ th subcarrier frequency to the half of the subcarrier bandwidth, respectively.

In the transmitter, the binary source-data sequence $b_{k}^{(\lambda)}$ of the $k$ th user is first spread by the random binary sequence $c_{k}^{(\hat{n})}$ with processing gain $N=T_{b} / T_{c}$, where $1 / T_{b}$ and $1 / T_{c}$ stand for the bit and chip rates, respectively, and $\lambda$ stands for the integer part of $\hat{n} / N$, i.e., $\lambda=\lfloor\hat{n} / N\rfloor$. Then, the spread signal, $b_{k}^{(\lambda)} c_{k}^{(\hat{n})}$, is shaped by a chip-waveform shaping filter with impulse and frequency responses $h(t)$ and $H(f)$, respectively. After that, the shaped signal modulates $M$ different subcarriers $\left\{\sqrt{2 P T_{c} / M} \cos \left(2 \pi f_{m} t+\theta_{m}^{(k)}\right)\right\}$, where $f_{m}$ and $\theta_{m}^{(k)}$ are the frequency and phase of the $m$ th subcarrier, respectively, and $P$ is the total power of all subcarrier-modulated signals. Finally, the $M$ binary phase-shift keying (BPSK) carrier-modulated signals are added together to form the transmitted signal. Note that when the required propagation distance is around $10 \mathrm{~m}$ for indoor UWB, for acceptable performance of a receiver, the total transmit power in a transmitter can be as small as $1 \mathrm{~mW}$. Therefore, the UWB transmitter will potentially not require a nonlinear power amplifier, thus avoiding the problem of intermodulation distortion among different subcarriers. In order to simplify the analysis, in any given subband, Gaussian narrowband interference with a flat spectrum, a total power of $J$, and a bandwidth of $B_{j}$, is assumed.

Some indoor UWB channel-measurement results have been reported in [7]. The UWB channels have the following characteristics.

1) At the receiver, a large number of resolvable paths can be observed, of which the number of paths within $10 \mathrm{~dB}$ of the peak power could be up to 40 .

2) Each path experiences less fading with respect to a conventional narrowband channel, where Rayleigh fading with relatively large fluctuation is usually implied, due to a large number of overlapping multipath components. However, UWB systems have a very high resolution in the time domain, so that only a small number of multipath components (including line-of-sight (LOS) and non-LOS) comprise each resolvable path. Therefore, a more appropriate fading model is Nakagami fading.

As a consequence, in this letter, we assume that the UWB channel is described by dense multipath Nakagami fading with the complex lowpass-equivalent impulse response

$$
h_{m}^{(k)}(t)=\sum_{l=1}^{L} \beta_{m, l}^{(k)}(t) \delta\left[t-\tau_{m, l}^{(k)}(t)\right] e^{j \phi_{m, l}^{(k)}(t)}
$$

where $L(L \geq 1)$ is the number of resolvable propagation paths in each subcarrier. For the sake of simplicity, all subcarriers of all users are assumed to have the same number of resolvable paths. In $(1), \phi_{m, l}^{(k)}(t)$ and $\tau_{m, l}^{(k)}(t)$ represent the phase and delay of the $l$ th path in the $m$ th subcarrier of the $k$ th user, respectively. It has been shown in [7] that the temporal correlation between adjacent paths is negligible. Therefore, all random variables in (1) are assumed to be independent of one another. The coefficient $\beta_{m, l}^{(k)}(t)$ represents the Nakagami fading component of the $l$ th path in the $m$ th subcarrier of the $k$ th user with probability density function (pdf)

$$
p\left(\beta_{l}\right)=2\left(\frac{\mu_{l}}{\Omega_{l}}\right)^{\mu_{l}} \frac{\beta_{l}^{2 \mu_{l}-1}}{\Gamma\left(\mu_{l}\right)} \exp \left(-\frac{\mu_{l}}{\Omega_{l}} \beta_{l}^{2}\right), \quad \beta_{l} \geq 0 .
$$

Assuming a slowly varying channel, $\beta_{m, l}^{(k)}(t), \phi_{m, l}^{(k)}(t)$ and $\tau_{m, l}^{(k)}(t)$ are taken as constant. In (2), $\Gamma(x)=\int_{0}^{\infty} t^{x-1} e^{-t} d t$ is the gamma function

$$
\Omega_{l} \hat{=} E\left\{\beta_{l}^{2}\right\}=e^{-(l-1) \delta}
$$

where $\delta$ is the rate of exponential decay of the multipath delay profile, and

$$
\mu_{l}=\frac{\Omega_{l}^{2}}{E\left\{\left(\beta_{l}^{2}-\Omega_{l}\right)^{2}\right\}}
$$

is the ratio of the square of the mean of $\beta_{l}^{2}$ to the variance of $\beta_{l}^{2}$, and stands for the fluctuation of the fading component $\beta_{l}^{2}$. When $\mu_{l}$ is large (small), the fading component is stable (diffuse). According to the measurement results in [7], the values of $\mu_{l}$ range between $6-1$, decreasing with increasing excess delay, and can be approximated as an exponential function of excess delay. This implies that multipath components arriving 


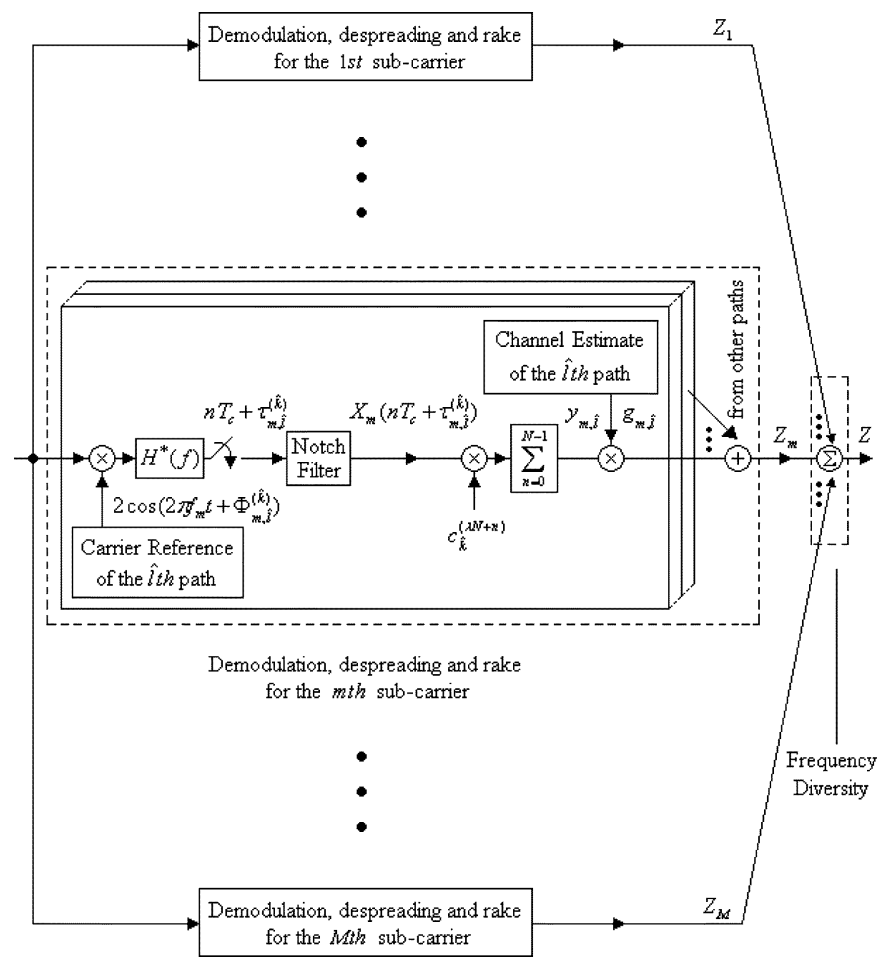

Fig. 2. Receiver for the $\hat{k}$ th user (reference user).

with long excess delays are more diffuse than are the first arriving components.

The multicarrier CDMA receiver is shown in Fig. 2 and consists of $M$ parallel branches, corresponding to $M$ subcarriers. Let us consider the $m$ th branch. The received signal is input to a frequency down-converter with a coherent carrier reference. For the $\hat{l}$ th path of the $\hat{k}$ th user, the reference is $2 \cos \left(2 \pi f_{m} t+\Phi_{m, \hat{l}}^{(\hat{k})}\right)$. After that, a baseband matched filter with frequency response $H^{*}(f)$ is employed. Basically, the frequency down-converter and the matched filter together translate the received RF signal to a baseband signal and remove out-of-subband additive white Gaussian noise (AWGN), and both narrowand interference and signals from other subcarriers. The output of the matched filter includes CDMA signals in the subcarrier band, in-band noise, and possibly narrowband interference. Here, at most one narrowband interferer is assumed in a given subcarrier band. Note that in order to have zero intersymbol interference (ISI), it is assumed that $X(f)=|H(f)|^{2}$ with inverse Fourier transform $x(t)$ satisfies the Nyquist criterion. The sampling time at the output of the matched filter corresponds to the $\hat{l}_{\text {th }}$ path of the $\hat{k}$ th user, i.e., $\tau_{m . \hat{l}}^{(\hat{k})}+n T_{c}$, where $n$ is an integer. Note that the $\hat{l}$ th path corresponds to one of the taps of the RAKE receiver. The baseband samples at the output of the matched filter are inputs to a digital notch filter (implemented as a double-sided digital Wiener filter with the number of taps of $W$ on each side and coefficients $\left\{\alpha_{w}^{(m)}, w=-W, \cdots, W\right\}$ where $\alpha_{0}^{(m)} \equiv 1$ ). While the notch filter causes ISI, or self-interference, to the desired multicarrier signal, this interference is typically negligible compared to multiple-access interference (MAI) [5]. The output of the notch filter is given by $X_{m}\left(n T_{c}+\tau_{m, \hat{l}}^{(\hat{k})}\right)$. Considering the $\hat{l}$ th path of the $\hat{k}$ th user, corresponding to the $\hat{l}$ th tap of the RAKE, the output of the notch filter will be despread by the random sequence $c_{\hat{k}}^{(\lambda N+n)}$ of the $\hat{k}$ th user, and the baseband signal $y_{m, \hat{l}}$ is obtained at the output of the accumulator.

The baseband signal $y_{m, \hat{l}}$ is weighted by the channel estimate $g_{m, \hat{l}}$ of the $\hat{l}$ th path to obtain the random variable $y_{m, \hat{l}} g_{m, \hat{l}}$. The channel estimate $g_{m, \hat{l}}$ is defined as the ratio of the desired signal amplitude to the variance of the noise and interference components in the output, $y_{m, \hat{l}}$, and is given by

$$
\begin{aligned}
g_{m, \hat{l}} & =\frac{E\left(y_{m, \hat{l}} \mid b_{\hat{k}}(\lambda)=1, \beta_{m, \hat{l}}^{(\hat{k})}\right)}{\sigma_{m, \hat{l}}^{2}} \\
& =\frac{E\left(y_{m, \hat{l}} \mid b_{\hat{k}}(\lambda)=1, \beta_{m, \hat{l}}^{(\hat{k})}\right)}{E\left\{y_{m, \hat{l}}-E\left(y_{m, \hat{l}} \mid b_{\hat{k}}(\lambda)=1, \beta_{m, \hat{l}}^{(\hat{k})}\right)\right\}^{2}} .
\end{aligned}
$$

It can be seen from (5) that when narrowband interference is still strong even after notching, a very small weighting factor $g_{m, \hat{l}}$ will be used, so that the weighted signal $y_{m, \hat{l}} g_{m, \hat{l}}$ becomes small. Combining all the RAKE tap outputs from all subcarriers, one obtains the final test statistic for the $m$ th subcarrier

$$
Z=\sum_{m=1}^{M} \sum_{\hat{l}=1}^{R} y_{m, \hat{l}} g_{m, \hat{l}}
$$

where $R(R \leq L)$ is the number of taps in the RAKE receiver.

\section{Performance AnAlysis}

As shown in Fig. 2 , considering the $\hat{l}$ th path of the $\hat{k}$ th user (reference user), corresponding to the $\hat{l}$ th finger of the RAKE, the accumulator output of the $m$ th branch is given by

$$
\begin{aligned}
y_{m, \hat{l}} & =\sum_{n=0}^{N-1} c_{\hat{k}}^{(\lambda N+n)} X_{m}\left((\lambda N+n) T_{c}+\tau_{m, \hat{l}}^{(\hat{k})}\right) \\
& \hat{=} A_{m, \hat{l}}+\sum_{\substack{k=1 \\
k \neq \hat{k}}}^{K} \mathrm{MAI}_{m, \hat{l}}^{(k)}+J_{m, \hat{l}}+N_{m, \hat{l}}
\end{aligned}
$$

where $A_{m, \hat{l}}$ is the desired signal component from the $\hat{l}$ th path of the $\hat{k}$ th user on the $m$ th subcarrier, corresponding to the center tap $(w=0)$ of the notch filter, and is given by

$$
A_{m, \hat{l}}=\sqrt{\frac{2 P T_{c}}{M}} \beta_{m, \hat{l}}^{(\hat{k})} b_{\hat{k}}^{(\lambda)} N .
$$

Note that for a large number of CDMA users (i.e., for $K \gg 1$ ), the effect of the multipath of the reference user is very small, since it roughly acts as one additional user. However, its inclusion in the analysis greatly complicates that analysis, and so it will be ignored. $\sum \mathrm{MAI}_{m, \hat{l}}^{(k)}$ is the MAI term from all the nonreference users in the $m$ th subcarrier with variance

$$
\begin{aligned}
\sigma_{\mathrm{MAI}}^{2}=\frac{P T_{c}}{M}( & K-1) \sum_{l=1}^{L} \Omega_{l} \sum_{w_{1}=-W}^{W} \sum_{w_{2}=-W}^{W} \alpha_{w_{1}}^{(m)} \alpha_{w_{2}}^{(m)} \\
& \times \sum_{n=0}^{N-1} \sum_{\hat{n}=0}^{N-1} R\left(w_{1}-\hat{n}+n, w_{2}-\hat{n}+n\right)
\end{aligned}
$$


where $R\left(n_{1}, n_{2}\right)$ is defined as

$$
\begin{aligned}
R\left(n_{1}, n_{2}\right) & =E\left\{x\left(\tau-n_{1} T_{c}\right) x\left(\tau-n_{2} T_{c}\right)\right\} \\
& =\frac{1}{T_{c}} \int_{0}^{T_{c}} x\left(\tau-n_{1} T_{c}\right) x\left(\tau-n_{2} T_{c}\right) d . \tau
\end{aligned}
$$

$J_{m, \hat{l}}$ represents the narrowband interference term with variance

$$
\begin{aligned}
\sigma_{J}^{2} & =E\left(J_{m, \hat{l}}^{2}\right) \\
& =\frac{J \cdot N}{p(1+\alpha)} \int_{\frac{(1+\alpha)(q-p)}{2}}^{\frac{(1+\alpha)(q+p)}{2}}\left|H_{w}\left(\frac{f}{T_{c}}\right)\right|^{2}\left|H\left(\frac{f}{T_{c}}\right)\right|^{2} d f \\
& =\frac{J \cdot N}{p(1+\alpha)} \hat{\sigma}_{J}^{2}
\end{aligned}
$$

where $H_{w}(f)$ is the frequency response of the notch filter, $H(f)$ is the frequency response of the matched filter, $\alpha$ is the rolloff factor of a raised-cosine filter, and $\hat{\sigma}_{J}^{2}$ is defined as

$$
\hat{\sigma}_{J}^{2}=\int_{\frac{(1+\alpha)(q-p)}{2}}^{\frac{(1+\alpha)(q+p)}{2}}\left|H_{w}\left(\frac{f}{T_{c}}\right)\right|^{2}\left|H\left(\frac{f}{T_{c}}\right)\right|^{2} d f .
$$

$N_{m, \hat{l}}$ is due to the thermal noise with variance of $\sigma_{N}^{2}=$ $N_{o} N \sum_{w=-W}^{W}\left(\alpha_{w}^{(m)}\right)^{2}$, where $N_{0}$ is the single-sided PSD of the white channel noise.

When $K$ is large, the total MAI term can be approximated as a Gaussian random variable. The narrowband interference and channel noise are Gaussian and are uncorrelated with the MAI term. Therefore, the total interference in (7) can be approximated by a Gaussian random variable. Thus, given $\beta_{m, \hat{l}}^{(\hat{k})}$, the signal-to-noise ratio (SNR) of $y_{m, \hat{l}}$, is given by

$$
\zeta_{m, \hat{l}}=\frac{\frac{2 P}{M} T_{b} N\left[\beta_{m, \hat{l}}^{(\hat{k})}\right]^{2}}{\sigma_{M R I}^{2}+\sigma_{J}^{2}+\sigma_{N}^{2}}=\gamma(m)\left[\beta_{m, \hat{l}}\right]^{2}
$$

where the sup " $k$ " is removed for simplicity of notation, and $\gamma(m)$ is the SNR, excluding the fading factor.
Now, combining all signals according to (6), the $\operatorname{SNR} \zeta$ can be written as

$$
\begin{aligned}
\zeta & =\frac{E^{2}\left\{Z \mid\left\{\beta_{m, \hat{l}}\right\}\right\}}{\operatorname{Var}\left\{Z \mid\left\{\beta_{m, \hat{l}}\right\}\right\}} \\
& =\sum_{m=1}^{M} \sum_{\hat{l}=1}^{R} \zeta_{m, \hat{l}} \\
& =\sum_{m=1}^{M} \gamma(m) \sum_{\hat{l}=1}^{R} \beta_{m, \hat{l}}^{2} \\
& =\gamma_{1} \sum_{m=1}^{M_{1}} \sum_{\hat{l}=1}^{R} \beta_{m, \hat{l}}^{2}+\gamma_{2} \sum_{m=M_{1}+1}^{M} \sum_{\hat{l}=1}^{R} \beta_{m, \hat{l}}^{2} \\
& \triangleq \Delta \gamma_{1} \zeta_{1}+\gamma_{2} \zeta_{2}
\end{aligned}
$$

where $M_{1}$ stands for the number of narrowband interference terms with $M_{1} \leq M$, and $\gamma_{1}$ and $\gamma_{2}$ are the SNRs of the subcarriers with and without narrowband interference, respectively, i.e., as shown in (15) at the bottom of the page, and

$$
\begin{aligned}
\gamma_{2} & =\frac{\frac{2 P}{M} T_{b} N}{\sigma_{\mathrm{MAI}}^{2}+\sigma_{N}^{2}} \\
& =\left\{\frac{K-1}{2 N^{2}} \sum_{l=1}^{L} \Omega_{l} \sum_{n=0}^{N-1} \sum_{\hat{n}=0}^{N-1} R(\hat{n}-n, \hat{n}-n)+\left(\frac{2 E_{b}}{N_{0}}\right)^{-1}\right\}^{-1} .
\end{aligned}
$$

Note that when narrowband interference is absent in the $m$ th subcarrier, a notch filter is not used (i.e., $\alpha_{w}^{(m)}=0, w \neq 0$ ). In (14), $\zeta_{1}$ and $\zeta_{2}$ are given by

$$
\zeta_{1}=\sum_{m=1}^{M_{1}} \sum_{\hat{l}=1}^{R} \beta_{m, \hat{l}}^{2}
$$

and

$$
\zeta_{2}=\sum_{m=M_{1}+1}^{M} \sum_{\hat{l}=1}^{R} \beta_{m, \hat{l}}^{2}
$$

Given $\left\{\beta_{m, \hat{l}}^{2}, m=1, \cdots, M, \hat{l}=1, \cdots, R\right\}$, the decision variable $Z$ can be approximated by a Gaussian random variable. The SNR is given by $\gamma_{1}$ when narrowband interference

$$
\begin{aligned}
\gamma_{1}= & \frac{\frac{2 P}{M} T_{b} N}{\sigma_{\mathrm{MAI}}^{2}+\sigma_{J}^{2}+\sigma_{N}^{2}} \\
= & \left\{\frac{K-1}{2 N^{2}} \sum_{l=1}^{L} \Omega_{l}\left\{\sum_{w_{1}=-W}^{W} \sum_{w_{2}=-W}^{W} \alpha_{w_{1}}^{(m)} \alpha_{w_{2}}^{(m)} \sum_{n=0}^{N-1} \sum_{\hat{n}=0}^{N-1} R\left(w_{1}-\hat{n}+n, w_{2}-\hat{n}+n\right)\right\}\right. \\
& \left.+\left(\frac{J}{P}\right)\left(\frac{M}{N}\right) \frac{1}{p(1+\alpha)} \hat{\sigma}_{J}^{2}+\left(\frac{2 E_{b}}{N_{0}}\right)^{-1} \sum_{w=-W}^{W}\left(\alpha_{w}\right)^{2}\right\}^{-1}
\end{aligned}
$$


is present in the $m$ th subcarrier, and is given by $\gamma_{2}$ otherwise. The conditional error probability, conditioned on $\left\{\beta_{m, \hat{l}}^{2}, m=\right.$ $1, \ldots, M, \hat{l}=1, \ldots, R\}$, is given by

$$
P_{e}(\zeta)=Q(\zeta)=\int_{\sqrt{\zeta}}^{\infty} \frac{1}{\sqrt{2 \pi}} \exp \left(-\frac{x^{2}}{2}\right) d x
$$

The average bit-error probability (BEP) $P_{e}$ can be obtained by averaging $P_{e}(\zeta)$ over $\zeta$, i.e.,

$$
\begin{aligned}
P_{e} & =\int_{0}^{\infty} P_{e}(\zeta) p(\zeta) d \zeta \\
& =\underbrace{\int_{0}^{\infty} \int_{0}^{\infty} \cdots \int_{0}^{\infty}}_{M \cdot R} P_{e}\left(\gamma_{1} \sum_{m=1}^{M_{1}} \sum_{\hat{l}=1}^{R} \beta_{m, \hat{l}}^{2}+\gamma_{2} \sum_{m=M_{1}+1}^{M} \sum_{\hat{l}=1}^{R} \beta_{m, \hat{l}}^{2}\right) \\
& \cdot \int_{0}^{p\left(\beta_{1,1}, \beta_{1,2}, \cdots, \beta_{M, R}\right) d \beta_{1,1} d \beta_{1,2}, \ldots, d \beta_{M, R}} \\
& \int_{0}^{\infty} P_{e}\left(\gamma_{1} \zeta_{1}+\gamma_{2} \zeta_{2}\right) p\left(\zeta_{1}, \zeta_{2}\right) d \zeta_{1} d \zeta_{2}
\end{aligned}
$$

where $p\left(\beta_{1,1}, \beta_{1,2}, \ldots, \beta_{M, R}\right)$ is the joint pdf of multipath fading amplitudes and $p\left(\zeta_{1}, \zeta_{2}\right)$ is the joint pdf of $\zeta_{1}$ and $\zeta_{2}$. Equations (19) and (20) give a complete bit-error rate (BER) performance evaluation of the multicarrier CDMA overlay for UWB systems. However, (20) is generally too complicated to be computed, and the pdf $p(\zeta)$ is unobtainable if the Nakagami fading parameters in each path and each subcarrier are to be chosen arbitrarily. In order to simplify the numerical evaluation, it is assumed that the Nakagami distribution in each path is such that the ratio $\mu_{\hat{l}} / \Omega_{\hat{l}}$ is the same for all paths. Since $\Omega_{l}$ is exponential, $\mu_{l}$ should be also exponential with the same exponent. This assumption is approximately true, based on the channel measurement results of [7], which show $\mu_{l}$ decreases with increasing excess delay. Therefore, it can be shown that $\zeta_{1}$ or $\zeta_{2}$ has the Gamma pdf [11]

$$
p\left(\zeta_{i}\right)=\left(\frac{\mu_{T}}{\Omega_{T}}\right)^{\mu_{T}} \frac{\zeta_{i}^{\mu_{T}-1}}{\Gamma\left(\mu_{T}\right)} \exp \left(-\frac{\mu_{T}}{\Omega_{T}} \zeta_{i}\right), \quad \zeta_{i} \geq 0, \quad i=1 \text { or } 2
$$

where

$$
\Omega_{T}= \begin{cases}\sum_{m=1}^{M_{1}} \sum_{\hat{l}=1}^{R} \Omega_{m, \hat{l}}=M_{1} \sum_{\hat{l}=1}^{R} \Omega_{\hat{l}}, & \text { for } \zeta_{1} \\ \sum_{m=M_{1}+1}^{M} \sum_{\hat{l}=1}^{R} \Omega_{m, \hat{l}}=\left(M-M_{1}\right) \sum_{\hat{l}=1}^{R} \Omega_{\hat{l}}, & \text { for } \zeta_{2}\end{cases}
$$

and

$$
\mu_{T}= \begin{cases}\sum_{m_{1}=1}^{M_{1}} \sum_{\hat{l}=1}^{R} \mu_{m, \hat{l}=M_{1}} \sum_{\hat{l}=1}^{R} \mu_{\hat{l}}, & \text { for } \zeta_{1} \\ \sum_{m_{1}=M_{1}+1}^{M} \sum_{\hat{l}=1}^{R} \mu_{m, \hat{l}}=\left(M-M_{1}\right) \sum_{\hat{l}=1}^{R} \mu_{\hat{l}}, & \text { for } \zeta_{2}\end{cases}
$$

and where $\Omega_{\hat{l}}$ and $\mu_{\hat{l}}$ are given by (3) and (4), respectively. Substituting (21) into (20), the average BEP $P_{e}$ is given by

$$
\begin{aligned}
P_{e} & =\int_{0}^{\infty} P_{e}(\zeta) p(\zeta) d \zeta \\
& =\int_{0}^{\infty} \int_{0}^{\infty} Q\left(\gamma_{1} \zeta_{1}+\gamma_{2} \zeta_{2}\right) \cdot p\left(\zeta_{1}\right) p\left(\zeta_{2}\right) d \zeta_{1} d \zeta_{2} .
\end{aligned}
$$

In the special case, when $M_{1}=0$ or $M_{1}=M$, the double integration in (24) reduces to a single integration. In summary, the error probability is given by

$$
P_{e}= \begin{cases}\int_{0}^{\infty} \int_{0}^{\infty} Q\left(\gamma_{1} \zeta_{1}+\gamma_{2} \zeta_{2}\right) & \\ \cdot p\left(\zeta_{1}\right) P\left(\zeta_{2}\right) d \zeta_{1} d \zeta_{2}, & M_{1} \neq 0, M_{1} \neq M \\ \int_{0}^{\infty} Q\left(\gamma_{1} \zeta_{1}\right) \cdot p\left(\zeta_{1}\right) d \zeta_{1}, & M_{1}=M \\ \int_{0}^{\infty} Q\left(\gamma_{2} \zeta_{2}\right) \cdot p\left(\zeta_{2}\right) d \zeta_{2}, & M_{1}=0 .\end{cases}
$$

\section{NUMERICAL RESUlTS}

In this section, the effects of different system parameters on the BER performance of the multicarrier CDMA overlay for UWB communications are investigated numerically. The channel fading is assumed to be Nakagami distributed, as noted in Section II. The multipath intensity profile $\Omega_{l}$ is $e^{-(l-1) \delta}\left(\Omega_{l}=e^{-(l-1) \delta}\right)$, where $\delta$ is the exponential decay rate, and in this letter is taken to be $\delta=0.2$. As described in Section II, the fluctuation parameter $\mu_{l}$ of the channel fading decreases with increasing excess delay, and its values range from 6 to 1 . Thus, $\mu_{l}$ is also assumed to be exponential, e.g., $\mu_{l}=4 e^{-(l-1) \delta}$. Therefore, the ratio $\mu_{l} / \Omega_{l}$ is set to be constant, i.e., $\mu_{l} / \Omega_{l}=4$ for all paths. The number of multipaths $L$ is 10 , the number of RAKE taps $R$ is six, the number of active users $K$ is 10 , the processing gain per subcarrier $N$ is 16 , the number of subcarriers $M$ is 10 , the ratio of narrowband interference bandwidth to the each subcarrier bandwidth $p=0.1$, the offset ratio of the center frequency of the narrowband interference from the corresponding subcarrier frequency to the half-subcarrier bandwidth $q=0.1$, and the number of taps on each side of the notch filters is $W=6$. The rolloff factor of the square-root raised-cosine filter is $\alpha=0.5$. Unless noted otherwise, $E_{b} / N_{0}=15 \mathrm{~dB}$.

In Fig. 3, the error probability of the multicarrier CDMA overlay, both with and without notch filters, is shown as a function of $E_{b} / N_{0}$. The ratio of narrowband interference power to the CDMA signal power $J / P=15 \mathrm{~dB}$, and the number of narrowband interferers $M_{1}=9$. For comparison, the simulation results are also shown. It can be seen that when $E_{b} / N_{0}$ is large, using notch filters can improve the overlay system performance by about one order of magnitude. Further, simulation and analytical results are very close.

Fig. 4 illustrates the system performance versus the number of narrowband interferers for various values of $J / P(0,5$, and $20 \mathrm{~dB})$. Note that, at most, one narrowband interferer is assumed in a given subcarrier. It can be seen from this figure that when the number $M_{1}$ of narrowband interferers is small (less than four), 


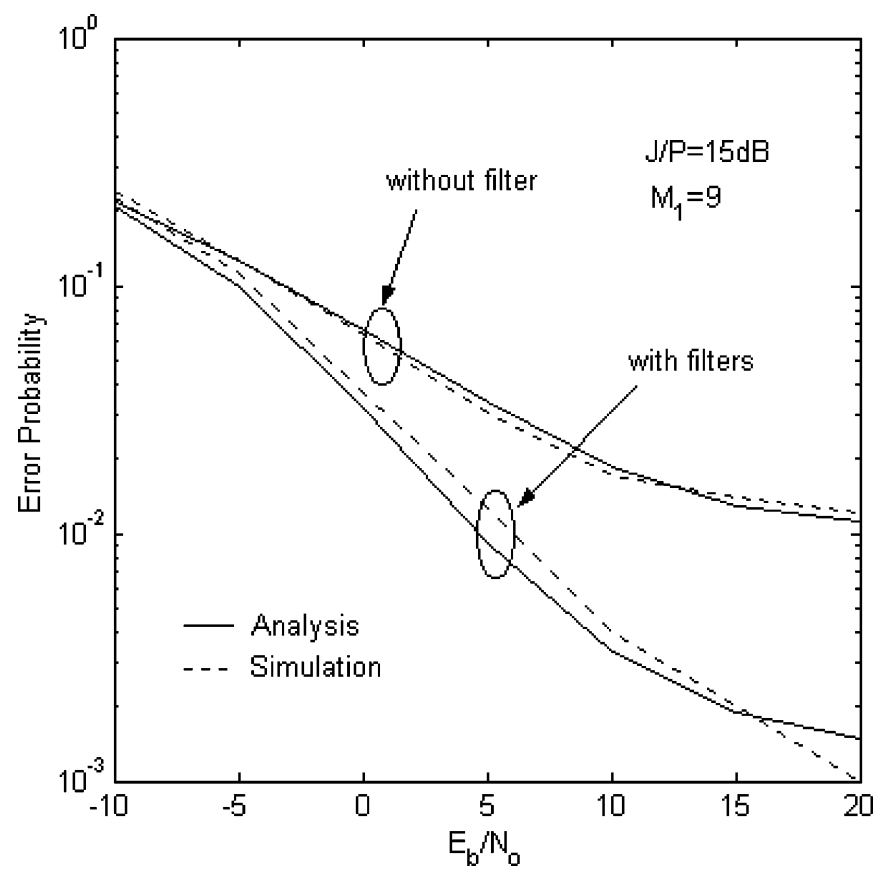

Fig. 3. Error probability as a function of $E_{b} / N_{0}$.

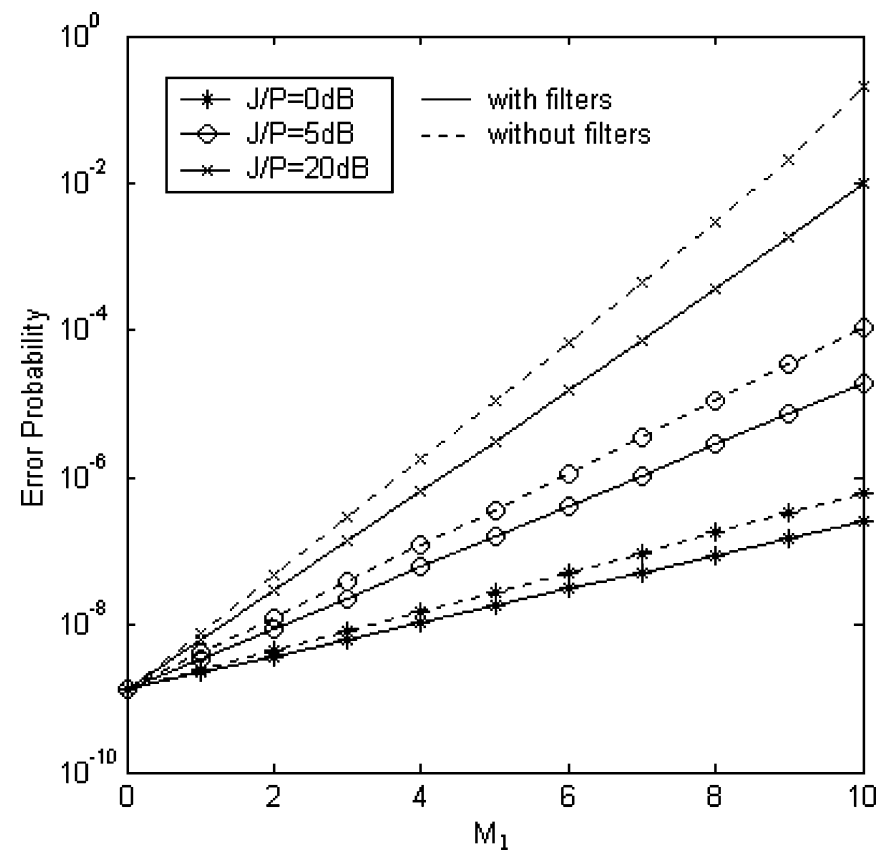

Fig. 4. Error probability versus the number of narrowband interferences.

system performance is good, due to frequency diversity from the unjammed subcarriers, irrespective of whether or not notch filters are used, and no matter how large $J / P$ is. However, when $M_{1}$ is large, performance with notch filters can be improved by an order of magnitude when $J / P$ is large.

Fig. 5 shows the error probability versus $J / P$ with and without notch filters for different values of $M_{1}$. It can again be seen that for a small value of $M_{1}$ (e.g., $M_{1}=2$ ), performance is satisfactory irrespective of whether notch filters are used. However, for a large value of $M_{1}$ (e.g., $M_{1}=M=10$ ), the performance gain with the notch filters is again seen to be sizeable.

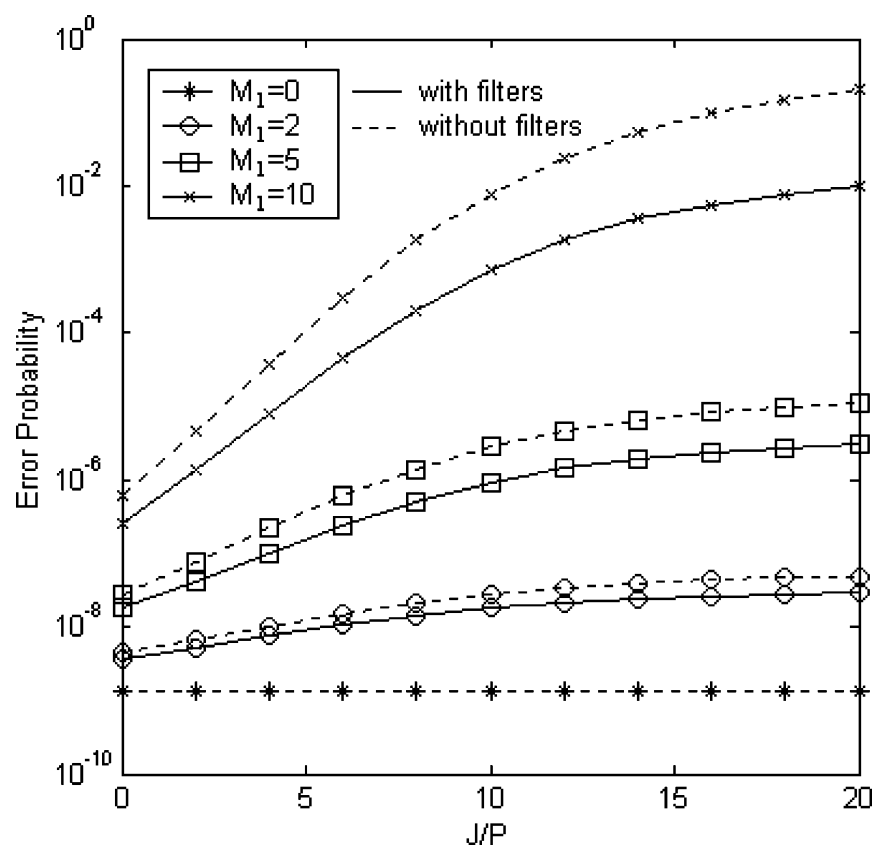

Fig. 5. Error probability versus the power ratio.

\section{CONCLUSIONS}

In this letter, a UWB system based upon multicarrier DS waveforms is studied in the presence of narrowband interference. It is shown that when the number of subcarriers jammed by narrowband interferers is small, the multicarrier receiver without notch filters can work well, due to the frequency diversity gain from nonjammed subcarriers. On the other hand, when the number of subcarriers jammed by the narrowband interferers is large, using notch filters can improve the multicarrier system performance significantly.

\section{REFERENCES}

[1] M. L. Welborn, "System considerations for ultra-wideband wireless networks," in Proc. Radio and Wireless Conf., 2001, pp. 121-124.

[2] T. Mitchell, "Broad is the way," IEE Rev., pp. 35-39, Jan. 2001.

[3] M. Win and R. A. Scholtz, "Ultra-wide bandwidth time-hopping spreadspectrum impulse radio for wireless multiple-access communications," IEEE Trans. Commun., vol. 48, pp. 679-691, Apr. 2000.

[4] L. B. Milstein et al., "On the feasibility of a CDMA overlay for personal communications networks," IEEE J. Select. Areas Commun., vol. 10, pp. 655-668, May 1992.

[5] J. Wang and L. B. Milstein, "CDMA overlay situations for microcellular mobile communications," IEEE Trans. Commun., vol. 43, pp. 603-614, Feb. 1995.

[6] S. Kondo and L. B. Milstein, "Performance of multicarrier DS-CDMA systems," IEEE Trans. Commun., vol. 44, pp. 238-246, Feb. 1996.

[7] D. Cassioli, M. Win, and F. Molisch, "The ultra-wide bandwidth indoor channel: From statistical model to simulations," IEEE J. Select. Areas Commun., vol. 20, pp. 1247-1257, Aug. 2002.

[8] W. Xu and L. B. Milstein, "On the performance of multicarrier RAKE systems," in Proc. IEEE GLOBECOM, Nov. 1997, pp. 295-299.

[9] D. Lee and L. B. Milstein, "Comparison of multicarrier DS-CDMA broadcast systems in a multipath fading channels," IEEE Trans. Commun., vol. 47, pp. 1897-1904, Dec. 1999.

[10] E. Sourour and M. Nakagawa, "Performance of orthogonal multicarrier CDMA in a multipath fading channel," IEEE Trans. Commun., vol. 44, pp. 356-367, Mar. 1996.

[11] E. K. Al-Hussaini and A. A. M. Al-Bassiouni, "Performance of MRC diversity systems for detection of signals with Nakagami fading," IEEE Trans. Commun., vol. COM-33, pp. 1315-1319, Dec. 1985. 